\title{
TENDENCIAS DE LA TRANSICIÓN DEMOGRÁFICA EN HONDURAS Y SU INCIDENCIA EN EL DESARROLLO
}

\author{
PhD. HÉCTOR YOBANY BACA SANTOS \\ hbdemografia@gmail.com \\ Tegucigalpa, Honduras
}

DOI: $10.5377 /$ rpdd.v4i1.9385

Recibido: abril, 2017

Aceptado: julio, 2017

\section{RESUMEN}

Los estudios demográficos son importantes porque permiten planificar nuevos programas, evaluar impactos, identificar problemas con proyección, etc. en beneficio de la población. En el desarrollo histórico los demógrafos han logrado determinar tres explicaciones (teorías) que han permitido conocer y entender el comportamiento del crecimiento de la población, estas explicaciones brindan planteamientos demográficos relevantes del mundo actual, tales como: teoría de la transición demográfica, teoría de la segunda transición demográfica y la teoría de la revolución reproductiva. Estas teorías buscan disminuir los riesgos sociodemográficos investigando, explicando y proyectando el comportamiento de la fecundidad, la mortalidad y el crecimiento demográfico. El comportamiento demográfico en Honduras, es que la fecundidad tiene una tendencia descendente, según proyecciones en término de dos décadas, la fecundidad en Honduras no llegará al nivel de reemplazo, lo que provocará el fin del bono demográfico y décadas después la entrada del invierno demográfico. La caída de la fecundidad está influida por la entrada de la posmodernidad, la cual está determinada por el comportamiento ascendente de las variables demográficas: Escolaridad, mercado laboral, uso de anticonceptivos, primera relacion sexual, etc. 


\title{
PALABRAS CLAVE
}

Demografía, postmodernidad, fecundidad, transición, familia.

\begin{abstract}
Demographic studies are important because they allow you to plan new programs, evaluate impacts, identify problems with projection, etc. For the benefit of the population. In the historical development of the demographers they have been able to determine three explanations (theories) that have to know and understand the behavior of population growth, these explanations, relevant demographic approaches of the real world, stories such as demographic transition theory, The second demographic transition and reproduction reproduction theory. These theories seek to reduce sociodemographic risks by investigating, explaining and projecting fertility behavior, mortality and demographic growth. The demographic behavior in Honduras is that fertility has a downward trend, according to projections within two decades, fertility in Honduras will not reach the replacement level, which will lead to the end of the demographic bonus and decades after the entry Of the winter Demographic The fall of fertility is influenced by the entry of postmodernity, which is determined by the upward behavior of the demographic variables: Schooling, labor market, contraceptive use, first sexual intercourse, etc. The indicators of these variables indicate that the demographic transition is affecting the postmodernity of Honduran families; It requires the attention of academics, government and all organizations that promote development to creating strategies or modyfing policies to address this future reality.
\end{abstract}

\section{INTRODUCCIÓN}

Los cambios que suceden en el mundo promueven el estudio y el análisis de diversos fenómenos, sus resultados permiten comprender de mejor manera la realidad y actuar para evitar sus consecuencias. El contenido de este artículo tiene como propósito explicar la incidencia de la transición demográfica en los patrones posmodernos de la familia hondureña y su relación con el desarrollo humano sostenible; explicar los cambios que ha tenido la familia en su organización interna como resultado de la transición demográfica y su incidencia en procesos de mejora de la calidad vida de la población, y establecer el nivel de posmodernidad de la sociedad hondureña, desde la perspectiva de los nuevos modelos de organización familiar. La nueva organización de la familia hace necesario el estudio de algunos indicadores socioeconómicos y demográficos que permiten identificar el nivel de posmodernidad y las condiciones en que se encuentra organizada la familia actual en la sociedad hondureña. 
La finalidad de este artículo es dar a conocer como la "Transición Demográfica en Honduras incide en la posmodernización de las familias"; en la medida que la transición demográfica incide en estas variables, la familia hondureña se va a considerar posmoderna. La familia posmoderna se caracteriza porque la mujer busca obtener más autonomía y libertad en las decisiones que le permiten potenciar su propio desarrollo y felicidad, evitan la procreación y el matrimonio, se preparan más académicamente y tienen una mayor participación en el mercado laboral.

Este artículo se estructura en cuatro grandes temas que pretenden explicar las tendencias demográficas de Honduras, se inicia explicando la importancia de la demografía en la planificación de programas de desarrollo; en el segundo apartado se explica la incidencia de la transición demográfica en la posmodernidad, tomando como base de estudio el nuevo pensamiento de la mujer; en el tercer apartado se explican los logros demográficos que ha obtenido Honduras actualmente; y por último se plantean las tendencias demográficas de Honduras en el presente siglo. Una de las principales contribuciones de esta investigación es determinar el grado de posmodernidad de la sociedad hondureña, el cual se elaboró mediante un cálculo matemático, que reveló una posmodernidad superior al 50\%. El principal indicador para este índice es la tasa de fecundidad, la cual según investigaciones del Instituto Nacional de Estadísticas de Honduras, su tendencia es al descenso, de tal manera que en las próximas décadas, andará bajo el nivel de reemplazo.

En Honduras las mujeres aun no han llegado a este tipo de pensamiento postmoderno, sin embargo, en unas décadas lo estarán manifestando; en algunos contextos como en las principales ciudades estos comportamientos se están presentando en el sentido que las jóvenes están postergando la idea de casarse, para lograr realizarse como profesionales, lo que ha llevado a reducir la fecundidad, tal situación significa un cambio en la dinámica demográfica.

\section{METODOLOGÍA}

En esta investigación se busca analizar el impacto de la transición demográfica en la posmodernización de la familia hondureña, para lo cual se utilizó una serie de variables, las cuales se les ha dado un enfoque de género femenino porque la posmodernidad es impulsado por un nuevo rol de la mujer en el escenario social, económico, cultural etc., las variables seleccionadas son las que se consideran tener mayor impacto para la realización de este estudio, las cuales se han investigado por departamento a nivel de país.

Las variables se han dividido en dos grupos; las del primer grupo son utilizadas para la determinación del índice de posmodernidad en Honduras, y las del segundo 
grupo para determinar el impacto de la transición demográfica en la posmodernidad; las variables del primer grupo son las siguientes: Años de escolarización femenina (utilizando la edad mediana), participación femenina en el mercado laboral, tasa de urbanización femenina, tasa de separación femenina, tasa de uniones consensuales, porcentaje de uso de anticonceptivos, y edad mediana de la primera relación sexual, y en el segundo grupo, solo se incluyó, las tasas de fecundidad. Con los datos de estas variables se hizo un cálculo matemático (regresión lineal), para determinar el índice y posteriormente establecer como la transición demográfica incide en ella.

La generación del nuevo conocimiento se hizo realizando una investigación documental, con fuentes secundarias (censo de población y vivienda y encuestas demográficas), para hacer un estudio en el que se pueda tener una descripción y un análisis de la organización familiar en Honduras. Obtenidos los resultados de país se unieron con los planteamientos teóricos ya establecidos. Al hacer esta fusión de resultados se hizo una contrastación teórica y así se determinó si Honduras está experimentando las características de la posmodernidad o aún se encuentra con rasgos de la modernidad.

\section{Importancia de la Demografía}

El estudio de la dinámica y evolución de la población son de gran relevancia para que los gobiernos, instituciones u organizaciones generen proyectos, planteen y tracen programas para el desarrollo de la población. Los indicadores que se generan con la demografía sobre educación, ingreso, estado civil, permiten conocer su estado y de esta manera generar estrategias para potenciarlas. "La demografía es una disciplina que articula fenómenos sociales con los movimientos que presentan las poblaciones humanas" (Damianovic, 2008, pág. 7), ofrece características esenciales, profundas y meticulosas sobre la población en su desarrollo espacial y temporal.

Los estudios que surgen de la demografía permiten determinar el estado de la población en lo relacionado con tamaño, estructura y distribución de la población. Además visualizan con sus cálculos matemáticos el comportamiento futuro de la población. 


\section{Principales Teorías de la Demografía}

Los demógrafos han determinado tres teorías, que brindan las explicaciones demográficas más relevantes en el mundo actual, tales como: teoría de la transición demográfica, teoría de la segunda transición demográfica y la teoría de la revolución reproductiva. Los cambios de la población están determinados por las variables demográficas: natalidad, mortalidad y migración. El papel de la transición demográfica consiste en disminuir los riesgos sociodemográficos: la alta fecundidad, la alta mortalidad, el alto crecimiento demográfico y la estructura de edades joven.

La teoría de la transición demográfica tiene como tesis hipotética que las poblaciones tienden a pasar por ciertas etapas de cambio demográfico determinado por la evolución de la mortalidad y la natalidad, como respuesta a las transformaciones sociales y económicas que trajo la modernización industrial, ya que desde fines del siglo XIX quedó establecido que los niveles de fecundidad y mortalidad de la población, más que por leyes biológicas, están condicionados por factores socioeconómicos, culturales, políticos y geográfico- sociales.

La primera teoría de la transición demográfica, presentada por CELADE (2000, pág. 1), describe diferentes fases: la primera es la transición incipiente, la segunda es la transición moderada, la tercera es la plena transición y la última es la transición avanzada; cada país se ubica en una etapa de estas transiciones, el lugar que ocupe va a depender de los niveles de mortalidad y fecundidad que imperen en su realidad. La realidad hondureña está pasando por un periodo de plena transición (natalidad moderada y mortalidad moderada a baja, crecimiento moderado al 2\%), Se espera que en el futuro Honduras logre llegar a una mejor posición en la transición.

La teoría segunda transición demográfica, sostiene que la sociedad entra en una nueva etapa de organización, debido al protagonismo de las mujeres en las diferentes esferas de la realidad, lo que se refleja en el comportamiento de las variables demográficas (fecundidad y mortalidad) las que tienen niveles bajos, tanto que la fecundidad no llegan al nivel de reemplazo; el fundamento de esta teoría consiste en explicar los cambios que se han dado en la composición de la familia y de las uniones familiares en el mundo de Europa Occidental y otros países desarrollados (Australia, Canadá, Estados Unidos y algunos países de Europa Oriental).

La teoría segunda transición demográfica fue planteada en 1986 por dos teóricos, Lesthaghe y D.J. Van de Kaa, su planteamiento se hizo a partir de lo que se observaba en la evolución demográfica de las sociedades de esta región en el año de 1960 (Tasa de fecundidad bajo el nivel de reemplazo). 
Y tercer y ultima teoría es la revolución reproductiva (eficiencia demográfica o eficiencia reproductiva) se refiere a la manera de como las personas logran su reemplazo, no tanto con la fecundidad, sino con el aumento de la supervivencia. El nivel de reemplazo de la población es de 2.1 hijos por mujer, a esto también se le denomina fecundidad de reemplazo. Según Pérez y Macinnes (2008, pág. 97) "El salto de eficiencia demográfica que constituye el núcleo de la revolución reproductiva tiene dos causas: el aumento en la proporción de personas que sobreviven hasta el final de las edades reproductivas y el hecho de que sus hijos tengan vidas aún más largas que sus progenitores."

\section{Comportamiento de las variables de posmodernidad}

$\mathrm{Al}$ analizar la incidencia de transición demográfica en la posmodernidad, en el contexto hondureño, se considera el análisis de cada una de las variables; una de ellas es la escolarización, el informe de desarrollo humano (2014), señala que en Honduras, la mediana de años de escolarización para la población es de 5.5 años, al analizarlo por sexo la población femenina tiene una escolarización de 5.3 años, la cual se considera muy baja al compararla con la de los países desarrollados (12.7 años), no obstante esta variable ha ido en ascenso.

Al estudiar esta variable por departamento (INE, 2013), se observa que existen diferencias entre los departamentos, hay algunos en los que la barra sobresale más que otros; los departamentos de Honduras que tiene las mejores tasas de escolaridad son Francisco Morazán (5.9 años), Islas de bahía (5.7 años) y Cortés (5.6 años) y los departamentos con menor años de escolaridad es Lempira (3.0 años) y Copán (3.1 años). Estas diferencias entre departamentos se concentran en el occidente de Honduras, lo cual posiblemente se deba por la falta de atención por parte del gobierno, se puede evidenciar en el hecho de que en esta región se concentran los niveles más bajos de pobreza, según los indicadores de desarrollo.

La escolaridad de la mujer es una variable muy importante en la posmodernidad porque dependiendo de su nivel, es posible se esté experimentando un cambio en su modo de pensar, en el sentido de no depender de un hombre al momento de decidir formar un hogar o tener más libertad y autonomía en sus decisiones; una mayor escolaridad lleva a tener mayor participación en el mercado laboral.

A pesar de que la mujer ha logrado mayor acceso al mercado laboral, la población hondureña todavía no supera los problemas de pobreza, lo que se puede evidenciar en las condiciones de vida que vive la gran mayoría de la población. Al estudiar las estadísticas sobre esta variable, muestran que la población total femenina de Honduras participa en un $25.4 \%$ en el mercado laboral, una participación muy 
baja, pero con tendencias al ascenso. A nivel de departamento, existen diferencias en la participación al mercado laboral, hay algunos en los que hay más participación que en otros; los departamentos de Honduras que tienen la mayor participación laboral femenina son Francisco Morazán (35.3\%) y Gracias a Dios (35.5\%), y los departamentos con menor participación son Lempira (10.6\%) y Santa Barbará $(15.3 \%)$.

La mayor escolaridad y la mejor participación de la mujer en el mercado laboral, pueden traer como consecuencia más libertad y autonomía. Esta situación hace que las mujeres cambien sus esquemas mentales, de tal manera que su vida la planifican de una manera diferente a la tradicional, piensa menos en contraer matrimonio y si lo hacen en muchos casos se divorcian o se separan, llevando a que las mujeres tengan menos hijos, y algunas hasta ni desean tenerlos. Thomas Burch (1976) en un análisis comparativo acerca del tamaño y la estructura de las familias de 54 países en el período 1945-1954 y 64 países en el período 1955-1963, observabó la tendencia a la conformación de hogares de menor tamaño.

La población hondureña también empieza a experimentar estos cambios en la reducción del número de hijos de las familias, pero más tardíamente, en los años setenta (del siglo XX), estos cambios son el resultado de una nueva forma de ver la realidad, posiblemente las mujeres tienen una mayor educación ${ }^{1}$, mayor participación en el mercado laboral ${ }^{2}$ y quieren tener menos responsabilidades de hijos ${ }^{3}$. Estos cambios se fortalecen con la irresponsabilidad de los padres y más aun con el maltrato de los hombres hacia las mujeres ${ }^{4}$, de tal manera que una mujer prefiere vivir sola y tomar las riendas de su vida por su propia cuenta y prefiere tener una pareja en cohabitación evitando procrear hijos, usando métodos de planificación familiar.

$\mathrm{Al}$ analizar la variable de separación esta muestra que a nivel de país el 4.5\% de las mujeres ha decidido separarse o divorciarse de su pareja y ha preferido vivir sola. Al estudiar por departamento, los datos revelan que no existen diferencias muy significativas en la tasas de separación de las parejas; los departamentos que tienen las mayores tasas de separación son Francisco Morazán (5.3\%), Cortés y Comayagua $(4.9 \%)$ y los departamentos con menor tasa de separación son Gracias a Dios $(2.4 \%)$ y Lempira (3\%).

1 En el año 2006, la población de Honduras tenía 4.2 años promedio de estudio, promedio que subió a 7.5 años, hasta el año 2013 , obteniendo una ganancia de 3.3 años. Al estudiarlo por sexo se observa que las mujeres tienen más años de estudio que los hombres

2 La tasa de participación en los ingresos del hogar en Honduras, tiene una tendencia ascendente, tanto por sexo como por área de residencia. La tasa de participación general en el año 2006 fué de 50.7\%, en el año 2013 fué de $54.3 \%$, alcanzando una ganancia de $3.6 \%$ de mayor participación. 3 Las preferencias de fecundidad de las mujeres en Honduras, señalan que en el año 2012, solo un $12.1 \%$ de las mujeres desea tener más hijos, mientras que el 32.6\% no lo desea, y más aún el 22.6\% decide esterilizarse. Una forma de cómo evitar la procreación es usando los métodos anticonceptivos, los que más usan estos métodos son las personas que han llegado o terminado la educación superior, el $78.1 \%$ lo usa.4 Las mujeres que se encuentran entre las edades de $45-49$ años son las que reciben más violencia intrafamiliar por parte del esposo (41.1\%), seguido por el grupo de edad de 25-29 años, el 39.6\% son violadas de alguna forma por su esposo. 
Contrariamente a las separaciones se encuentran las uniones consensuales (parejas que viven en cohabitación); en Honduras no todas las parejas llegan a formalizar la unión del matrimonio, muchas solo llegan a unirse consensualmente, es decir la pareja (hombre y mujer) ha aceptado un status conyugal inferior al que se reconoce legalmente y vivir maritalmente en convivencia. Según el INE (2013), del total de las parejas en Honduras, el 27.18\% viven en cohabitación, de estas el 52\% son mujeres; a nivel de departamento el comportamiento de las uniones consensuales, los datos revelan que los dos departamentos que tienen las tasas más altas de parejas que viven en cohabitación son El Paraíso (32.13\%) y Gracias a Dios (31.54\%); y los dos departamentos que tienen las tasas más bajas son Intibucá (22.66\%) y La Paz (22.13\%).

El comportamiento del índice de posmodernidad en Honduras transita en un nivel aceptable, sus indicadores muestran que andan más cercanos a uno que ha cero. De las variables seleccionadas para determinar el índice posmodernidad, la que tiene el mejor índice de logro es la primera relación sexual (84\%), seguido del índice de uso de anticonceptivos, el cual a logrado un $79 \%$. los que tienen el menor índice logro son la escolaridad (24\%) y la participación femenina en el meracdo laboral (39\%).

Al relacionar el índice de postmodernidad con la transicion demográfica se afirma que esta tiene un impacto en los patrones posmodernos de la familia hondureña aunque no en un mayor grado, debido a sus pocos logros y avances en las variables seleccionadas.

Para determinar en Honduras el impacto de la transición demográfica en la posmodernidad de las familias, se analiza la fecundidad como la variable que se relaciona directamente con los indicadores de la posmodernidad. Mediante un modelo de regresión lineal se calcula la incidencia que tiene esta variable en la posmodernidad.

En la gráfica No. 1, se observa la incidencia de la fecundidad en la posmodernidad, se estima que el grado de asociación lineal entre ambas variables es media ya que anda por el $43 \%$, además se puede explicar que los cambios en la fecundidad inciden muy ligeramente en los cambios que se dan en el índice de posmodernidad, debido a que el $18 \%$ de la variación de los datos, es explicable con la recta de la regresión lineal, el $82 \%$ es explicable por errores aleatorios. Al analizar la relación entre las tasas de fecundidad y el índice de posmodernidad de cada departamento de Honduras, se aprecia que la linealidad de la fecundidad tiene una tendencia al descenso; y su dispersión sigue la misma tendencia, aunque sus datos no están concentrados debido a bajo nivel de variación de datos, lo que significa que la correlación entre la fecundidad y el índice de posmodernidad es negativa, (es decir a medida desciende la fecundidad el índice de posmodernidad asciende). 
Si la linealidad de la fecundidad fuese contraria a la dispersión de los datos, la correlación sería positiva, es decir a medida la fecundidad disminuye también lo haría la posmodernidad.

\section{Gráfico No. 1}

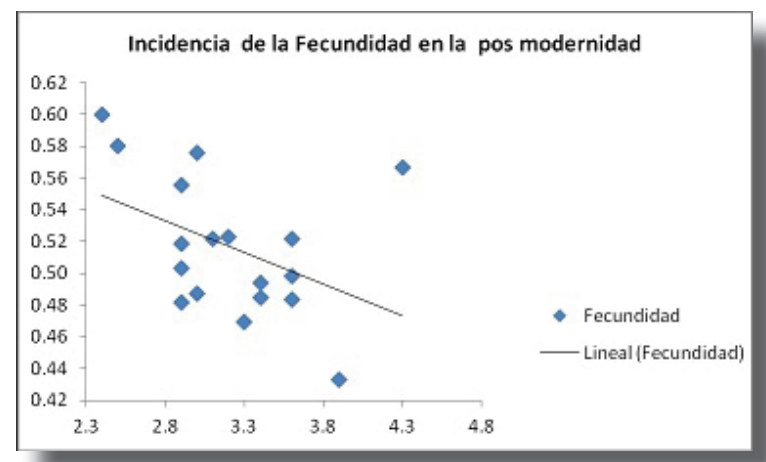

Fuente: Elaboración propia con datos de Instituto Nacional de Estadísticas. Honduras.

El análisis concluye señalando que el coeficiente de intercepción de la fecundidad, influye en los índices de posmodernidad, lo que se evidencia en sus valores estadísticos $\mathrm{t}$, los cuales demuestran ser estadísticamente significativos, ya que su valor es de 3.5\%. (Cuando el valor de t para el coeficiente es igual o superior a 2.0, significa que los valores son estadísticamente significativos; mientras más alto es este valor la incidencia de esta variable es mayor en la posmodernidad de las familias).

Del mismo modo, al analizar la incidencia de la fecundidad sobre las variables de la posmodernidad (uniones consensuales de la mujeres, uso de anticonceptivos por parte de la mujer, mediana de la primera relación sexual, mercado laboral y separaciones de las mujeres) su linealidad también es negativa en cada una de las variables, porque a medida la fecundidad desciende las tasas de las variables de la posmodernidad ascienden.

Al señalar que esta correlación entre la fecundidad y las variables de la posmodernidad es negativa, significa decir que el cambio en la reducción de la fecundidad incide en el aumento de la posmodernidad de las familias hondureñas.

\section{Tendencias de la transición demográfica en Honduras}

Cada país tiene una posición en esta transición, la que depende de los niveles de fecundidad y mortalidad, mientras más elevados andan estos niveles la transición es baja y lenta; por el contrario, si los niveles andan bajos su transición es alta y 
rápida. En el caso de Honduras tanto la fecundidad como la mortalidad han mejorado, aunque en las últimas décadas la mortalidad ha ido con menor ritmo.

La mortalidad en Honduras no ha tenido los resultados esperados, sus niveles aun andan altos, muy por arriba de la mediana centroamericana, lo mismo sucede con la mortalidad infantil, mortalidad neonatal y mortalidad materna, sin embargo se han reducido. En cambio la fecundidad si ha bajado su nivel, la mujer desea tener pocos o ningún hijo, algunas no desean tenerlos, por lo que usan algún método de planificación y otras buscan esterilizarse. Estos cambios en la fecundad están provocando que la eficiencia reproductiva descienda.

La realidad hondureña está pasando por un periodo de transición moderada (alta natalidad y moderada, y mortalidad moderada), la mortalidad se ha reducido a 4.8 por mil nacidos vivos, la esperanza de vida ha aumentado a 74.9 años y la Tasa Global de Fecundidad ha bajado a 2.9 hijos por mujer. Según proyecciones, sin las condiciones siguen igual la TGF bajará a 2.2 en el año 2025, casi el nivel de reemplazo, ${ }^{5}$ por lo que Honduras estaría llegando a una transición moderada alta. Estos cambios de la transición demográfica requieren la atención urgente del gobierno y de los académicos, para evitar sus efectos, porque perturba directamente a la producción del país, en el sentido que la población joven va hacer un número muy reducido, en contraste con la población adulta que va en aumento.

Estos cambios están correlacionados con las transformaciones que se manifiestan en todo el mundo, los cambios en los patrones del matrimonio y de la formación de una unión consensual ha debilitado la relación entre matrimonio y nacimiento de los hijos. Según la ONU (2014, pág. 8), en los últimos 20 años, los nacimientos fuera del matrimonio han aumentado como proporción del total en muchos países con un nivel de fertilidad bajo e intermedio. Los países que están experimentando estos cambios se encuentran en un grado alto de posmodernidad.

Los fundamentos de que Honduras está entrando en la posmodernidad es observable en el comportamiento de los indicadores socioeconómicos (escolaridad, participación en el mercado laboral, urbanización, divorcios y separaciones, uniones consensuales, edad mediana de la primera relación sexual y uso de anticonceptivos) y sociodemográficas (fecundidad).

5 Según la Transición demográfica todos aquellos países que se encuentren con una TGF entre las tasas $3.5>\mathrm{TGF}>2.5$ y Esperanza de Vida al nacer entre los años $71<$ Eo $<76$, están pasando por una transición moderada, si los niveles andan por arriba de estas cifras tienen una transición moderada alta, y si andan bajo estas cifras tienen una transición baja 
Los indicadores de estas variables señalan que la transición demográfica está incidiendo en la posmodernidad de las familias hondureñas, casi en un $60 \%$; lo cual explica que la tendencia descendente de la fecundidad está dando como resultado una tendencia ascendente de las variables de la posmodernidad. Según estimaciones en Honduras para el año 2030, la tasa de fecundidad va andar muy por debajo del nivel de reemplazo, lo que significa la entrada del invierno demográfico; se requiere mejorar las condiciones de vida de la población en lo referido a una mejor educación y salud (en cobertura y calidad), mejores empleos (mejores pagos y más estables) y mejores sistemas de seguridad social (mejor atención en salud y mejores sistemas de jubilación).

\section{CONCLUSIONES}

- La revolución reproductiva explica el cambio demográfico que están experimentando el mundo desarrollado y que empezará a manifestarse en las próximas décadas en el resto de los países, entre ellos Honduras y los de América Latina. La base explicativa de esta revolución se fundamenta en los cambios en la estructura de la familia y la reducción del número de hijos, lo que podría deberse a una serie de causas como la mayor participación de la mujer en la vida económica, política y social.

- En Honduras el bono demográfico se espera finalice en la década de 2050 cuando aumente el número personas adultas mayores, respecto a los adultos en edad de trabajar. Al aumentar la dependencia de los adultos mayores de 60 años, ocasionaría un impacto negativo en la economía porque el número de consumidores aumenta y el de los productores decrece, además por los costos elevados en la atención a su salud; se entraría a una etapa de desventaja demográfica, situación que la economía de Honduras no podría sostener, con las condiciones que existen actualmente.

- Los cambios demográficos que se están manifestando en el contexto hondureño son el resultado de la revolución anticonceptiva y la mutación de valores derivada de los procesos de modernización social y económica, también se debe a las nuevas tendencias demográficas en el mundo por efecto de la globalización, influidas por la actuación de los canales simbólicos, los centros educativos, y los medios masivos de comunicación, los que han mediado en el pensamiento de la mujer.

- En Honduras las mujeres no han logrado llegar a este tipo de pensamiento (posmoderno), sin embargo, en unas décadas lo estarán manifestando; en algunos contextos como en las principales ciudades estos comportamientos se están 
presentando en el sentido que las jóvenes están postergando la idea de casarse, para lograr realizarse como profesionales, lo que ha llevado a reducir la fecundidad, tal situación significa un cambio en la dinámica demográfica.

- Ante la nueva dinámica demográfica actual y futura, los países como Honduras y los de la región latinoamericana, tienen una ventaja favorable para impulsar el desarrollo, el cual consiste en el aprovechamiento del bono demográfico, generando inversiones productivas, para generar empleo seguro y bien remunerado, aumentar la inversión social para mejorar la salud, la educación y reducir los índices de pobreza y así disminuir la inseguridad socioeconómica, para anticipar los costos de las demandas de la población adulta mayor. Se requiere que en Honduras se promuevan cambios en las esferas política, económica y social, para lograr el desarrollo de toda la sociedad en general.

\section{BIBLIOGRAFÍA}

Burch, T., Lira, L. y López, V. (1976) La familia como unidad de estudio demográfico.

Centro Latinoamericano de Demografía. Serie E, No. 1001. San José, Costa Rica.

Centro Latinoamericano de Demografía (2000) La segunda transición demográfica: riesgos sociodemográficos, capacidad de respuesta y Mecanismos de adaptación.

Capítulo IV tomado de CEPAL/CELADE. http://www.eclac.org/publicaciones/ xml/4/10264/LCR2086_IV.pdf

Damianovic, N. (2008) Población y sociedad, aspectos demográficos, 1950 - 2004. Publicación Especial. Instituto Nacional de Estadísticas, Departamento de Estadísticas Demográficas y Vitales, Departamento Imagen Corporativa. Santiago, Chile.

Instituto Nacional de Estadísticas (2013) XVII Censo Nacional de Población y VI de Vivienda. Tegucigalpa, Honduras.

Instituto Nacional de Estadísticas (2011-2012) Encuesta Nacional de Demografía y Salud (ENDESA). Tegucigalpa, Honduras.

Organización de Naciones Unidas (2014) Situación demográfica en el mundo (informe conciso). Departamento de Asuntos Económicos y Sociales División de Población. 
Nueva York. Pérez, J., MacInnes J. (2008) La tercera revolución de la modernidad; la revolución reproductiva. Universidad de Edimburgo. Instituto de Economía y Geografía. Revista Española de Investigaciones Sociológicas (Reis) N. ${ }^{\circ} 122$.

Programa de las Naciones Unidas Para el Desarrollo (2014) Informe sobre Desarrollo Humano: Sostener el Progreso Humano: Reducir vulnerabilidades y construir resiliencia. Impreso en Estados Unidos. 1 UN Plaza, Nueva York, NY 10017, Estados Unidos. 